\title{
Courtney Coursey Moreno and Pardeep Kumar Mittal (Eds.), Gastrointestinal imaging: a teaching file
}

\author{
Wolters Kluwer, Philadelphia, 2014, 192 pp
}

\author{
Kristina Flicek \\ Department of General Surgery, Mayo Clinic Hospital, Scottsdale, AZ 85259, USA
}

The intent of this book is to provide a resource for trainees learning the basics of gastrointestinal imaging (fluoroscopy, CT, and MRI) as well as a review aid when studying for certifying examinations. This case-based book includes 300 teaching files divided between the three different modalities. The format of the book aims to walk the reader through a step-by-step process of how to work through unknown cases by providing only a brief clinical history and images. Differential diagnoses, final diagnosis, and an explanation of the case are also offered for each case.

This review book contains 51 fluoroscopy, $27 \mathrm{CT}$, and 22 MRI cases. All chapters begin with one line of clinical history (patient age, symptom, and any pertinent past medical or surgical history). One or more images are shown, and often more than one imaging modality (i.e., ultrasound) is included within the CT and MRI sections. A list of two or more differential diagnoses is given, and the actual diagnosis is listed below. A discussion follows which includes a brief paragraph for each of the differential diagnoses including etiology and other pertinent facts about the disease process. For each case, there are also additional questions with answers and a section which lists a few points that are important for radiologists to communicate to the ordering physician.
Overall, this text is clearly written and offers a lot of information in a concise format. The images provided are clear and adequately illustrate the disease process. One suggestion in the future to make this book even stronger is to provide additional pictures illustrating the differential diagnoses. This side by side comparison would help the readers visualize the differences between the diagnoses and make the teaching extremely thorough. In addition, showing the same images with arrows or figures, or in comparison to a normal image at the end of the case would help illustrate the abnormalities for less experienced trainees.

This reference is ideally suited for trainees including medical students, residents, and fellows. This book would provide a good foundation for common diseases identified with gastrointestinal imaging including fluoroscopy, MRI, and CT. I would recommend this text to medical students, residents, and fellows who are being exposed to their first rotation in gastrointestinal imaging or are reviewing for their certifying exams. Dr. Moreno and Mittal have produced a high-quality review text and have adequately fulfilled their objectives. 